Fabrication of diffractive optical elements using the CMOS process

This article has been downloaded from IOPscience. Please scroll down to see the full text article.

2002 J. Micromech. Microeng. 1221

(http://iopscience.iop.org/0960-1317/12/1/304)

The Table of Contents and more related content is available

Download details:

IP Address: 140.112.113.225

The article was downloaded on 19/12/2008 at 07:59

Please note that terms and conditions apply. 


\title{
Fabrication of diffractive optical elements using the CMOS process
}

\author{
Ching-Liang Dai ${ }^{1}$, Hung-Lin Chen ${ }^{2}$, Chi-Yuan Lee ${ }^{2}$ and \\ Pei-Zen Chang ${ }^{2}$
}

${ }^{1}$ Department of Mechanical Engineering, Oriental Institute of Technology, Taipei, 220,

Taiwan, Republic of China

${ }^{2}$ Institute of Applied Mechanics, National Taiwan University, Taipei, 107, Taiwan,

Republic of China

E-mail: fd032@ica.oit.edu.tw

Received 5 June 2001, in final form 25 October 2001

Published 11 December 2001

Online at stacks.iop.org/JMM/12/21

\begin{abstract}
In this paper we propose a novel diffractive optical element (DOE) which is fabricated by the conventional complementary metal-oxide semiconductor (CMOS) process. A simple post-CMOS process is employed to establish the relief pattern. The pattern can be used directly for its optical properties or can serve as a mould for subsequent replication. In this study we design a DOE with eight-phase-level gratings, which is compatible with the CMOS process. The eight-phase-level gratings have $81.4 \%$ diffraction efficiency calculated by computational and theoretical analyses. The experiment finished four-phase-level gratings.
\end{abstract}

\section{Introduction}

Rapid advances in diffractive optical technology have led to high performance and compact optical systems. Diffractive optical elements (DOEs) can perceptibly minimize the optical system and satisfy some particular demands more effectively than traditional optical elements. Hybrid lenses, thin lenses, wavefront correctors, wavefront generators, beam samplers and lens arrays can be improved by DOEs. Much research has fallen into three categories: design [1]; pattern generation [2]; and pattern replication [3]. The shape of the pattern should first yield high diffractive efficiency. The pattern is fabricated by various methods to satisfy the requirements. Replication is frequently used for low cost and high volume industrial production processes.

The design of DOEs should correspond to the theoretical constraints that determine the shape. The scalar theorem is usually used to predict the diffraction efficiency, but it provides only an approximate and overly optimistic solution. In contrast, the rigorous electromagnetic theorem is more precise, but complicated. The extended scalar theorem, however, adds a degree of intuition to understanding how the solutions vary, and suggests how exact solutions are calculated by commercial computer software according to the rigorous electromagnetic theorem.

The relief pattern can be performed in various ways according to the design, including grey-level masking [4], half-tone masking [5], bulk micromachining [6], electron beam writing [7], laser beam writing and the LIGA [8] process. Table 1 summarizes the advantages of each process.

The technology for replicating DOEs has achieved high resolution. Nanometre-sized microstructures, over areas of many square centimetres, can be manufactured by casting, embossing and moulding. DOEs are most economically mass produced by replication.

Many microsensors [9-12], microactuators [13, 14] and microstructures [15-17] have been fabricated by the complementary metal-oxide semiconductor (CMOS) process. The advantage of this method is that micromachined devices can integrate with circuits in a monolithic chip [18], which reduces volume and cost.

The novel type of DOE proposed herein is adapted to the conventional CMOS process, and the scale is minimized by microfabrication. Only a simple post-CMOS process with plasma etching is required to transfer the accurate relief pattern. This pattern can be used directly for its optical properties or serve as a mould in subsequent replication. The configuration of the component and the diffractive efficiency are also presented.

\section{Device design}

The diffraction efficiency ultimately determines whether the DOEs will work in particular applications. The diffraction 
Table 1. Various methods for fabricating DOEs.

\begin{tabular}{|c|c|c|}
\hline Fabrication methods & Advantages & Process \\
\hline Grey-level mask & $\begin{array}{l}\text { Continuous profile and } \\
\text { only one mask is needed }\end{array}$ & Photolithography and dry etching \\
\hline Half-tone mask & Only one mask is needed & Photolithography \\
\hline Bulk micromachining & Forms a special blazed angle & Wet etching \\
\hline Electron beam writing & Fine features & Electron beam lithography \\
\hline Laser beam writing & Fine features and cheaper equipment & Laser beam lithography \\
\hline LIGA & High numerical aperture & Thermal treatment \\
\hline
\end{tabular}

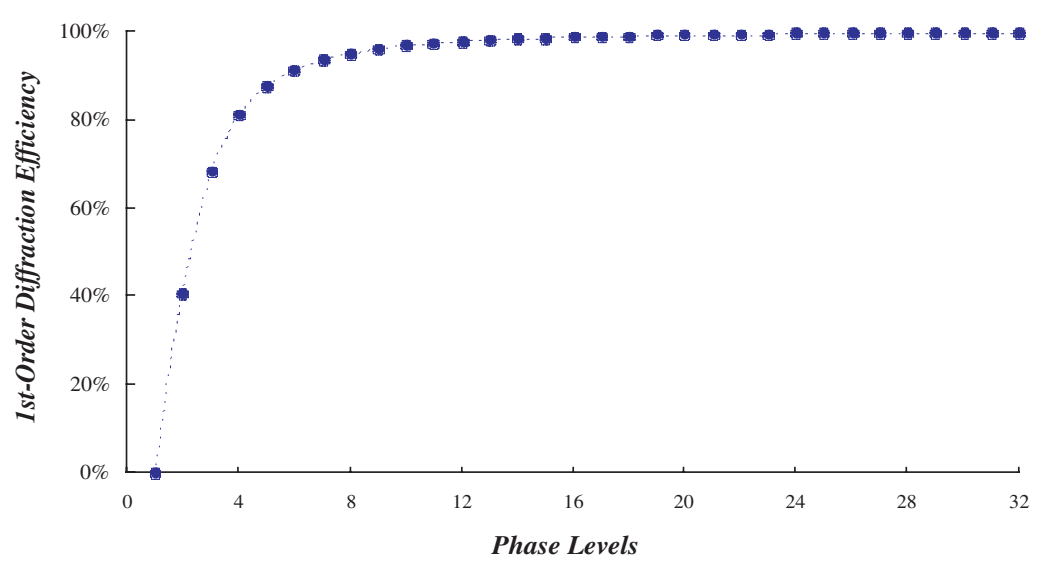

Figure 1. First-order diffraction efficiency versus phase levels calculated by scalar theory.

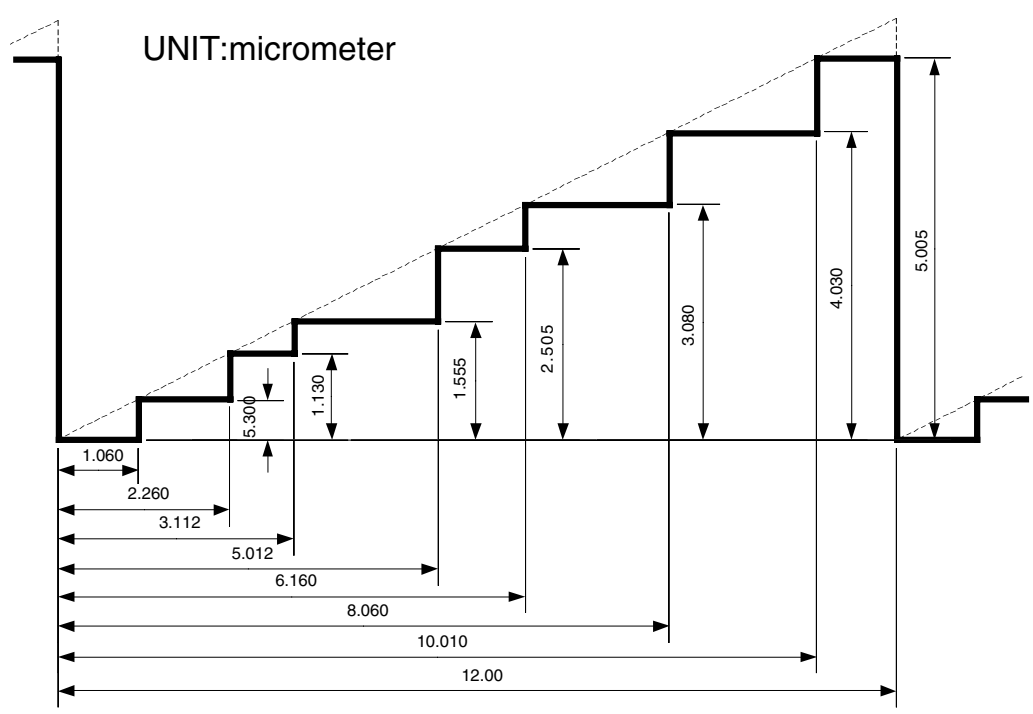

Figure 2. The shape and size of the model built in GSOLVER.

efficiency of arbitrary DOEs is directly influenced by the diffraction efficiency of the gratings. According to the scalar theory, the first-order diffraction efficiency $\eta$ of an optimized $N$-level grating can be expressed as [1]

$$
\eta=\left[\frac{\sin (\pi / N)}{\pi / N}\right]^{2} .
$$

The relationship between the diffraction efficiency $(\eta)$ and the phase levels $(N)$ is thus determined and shown as the plot in figure 1, which shows that four- and eight-phase-level gratings could reach $81 \%$ and $93 \%$ diffraction efficiencies, respectively.

Four- and eight-phase-level gratings have actually been designed here, corresponding to the conventional CMOS process. Since the conventional CMOS process is standardized and unchangeable, the essential constraint on the design of DOEs is the unequal depth of each phase level. The diffraction efficiency of the design thus cannot be merely calculated by the scalar theory, which represents the optimal case. Therefore, a real model of the design should be built and calculated.

A suitable size corresponding to the CMOS process is specified to yield high diffraction efficiency, as shown in figure 2. The model was also found by the computer software, GSOLVER, which is based on rigorous vector coupled-wave analysis and employed to analyse the various solutions. Then, the analytical equation derived from the extended scalar theory is used to verify the computational result. 


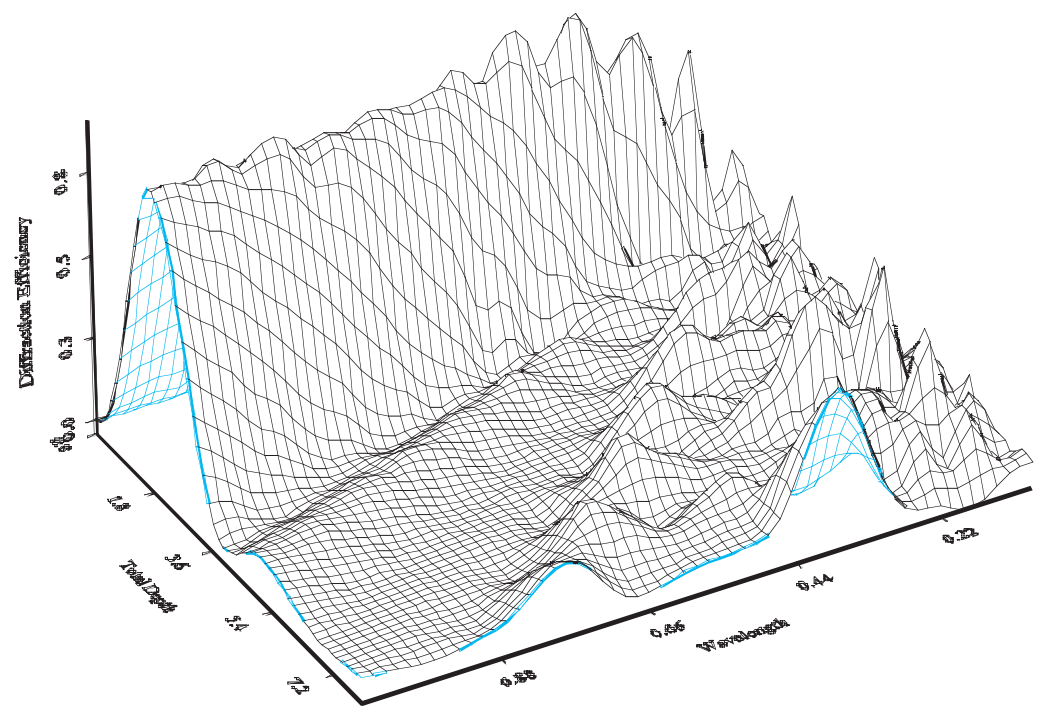

Figure 3. The first-order diffraction efficiency computed by GSOLVER with various depths and wavelengths.

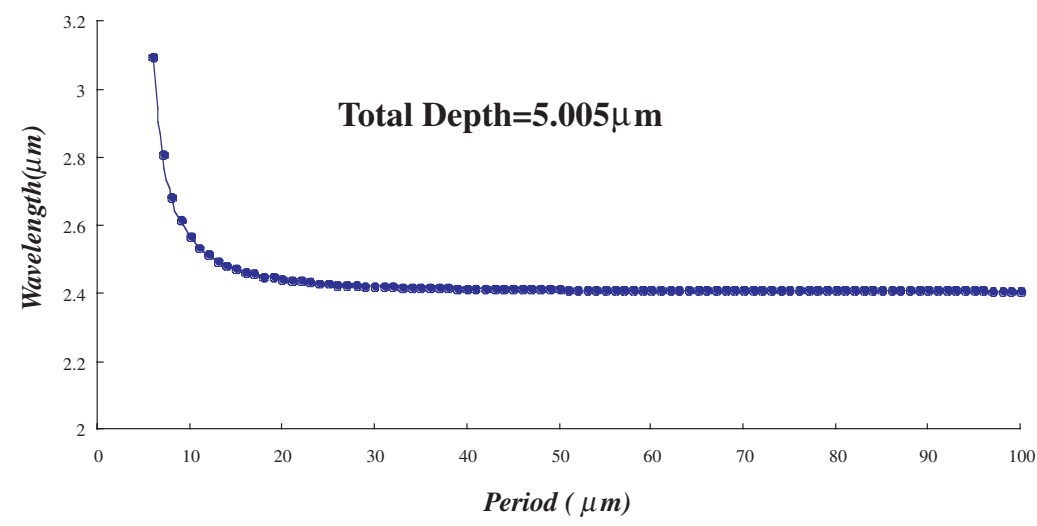

Figure 4. The optimum wavelength for various periods when the depth equals $5.005 \mu \mathrm{m}$.

In the model shown in figure 2, the eight-phase-level grating built in GSOLVER is assumed to have an index and period equal to 1.5 and $12 \mu \mathrm{m}$, respectively. The dashed line shows the original continuous gratings for which the brazed angle is $35.7^{\circ}$. The width is modified to fit the dashed line since the heights of each layer are constant and different from each other in the CMOS process. Figure 3 shows the computational result of first-order diffraction efficiency with various wavelengths of incident light, perpendicular to the substrate. The maximum diffraction efficiency is $81.4 \%$ at a $2.531 \mu \mathrm{m}$ wavelength, and the efficiency is high enough to be useful.

According to the extended scalar theory, the optimum grating profile depth can be written as [1]

$$
d=\frac{\lambda}{n-\sqrt{1-(\lambda / T)^{2}}}
$$

where $d$ is the depth, $\lambda$ is the wavelength, $n$ is the index, and $T$ is the period of the optimum grating profile. We let $d$ and $n$ equal $5.005 \mu \mathrm{m}$ and 1.5, respectively. Figure 4 plots the value of $T$ as a function of $\lambda$. The result is more precise over a longer period, because equation (2) is valid only for diffractive structures with very large period-to-wavelength ratios. When $T$ and $d$ equal (a)

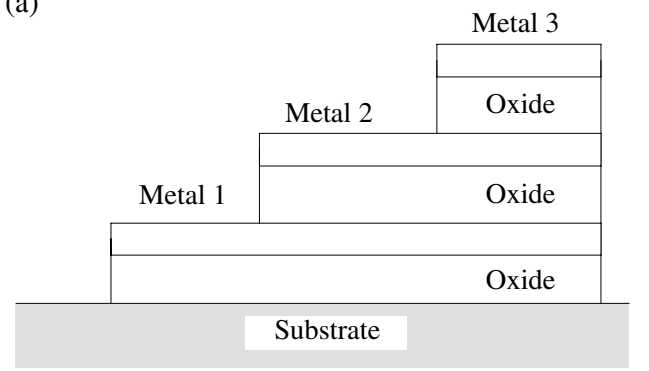

(b)

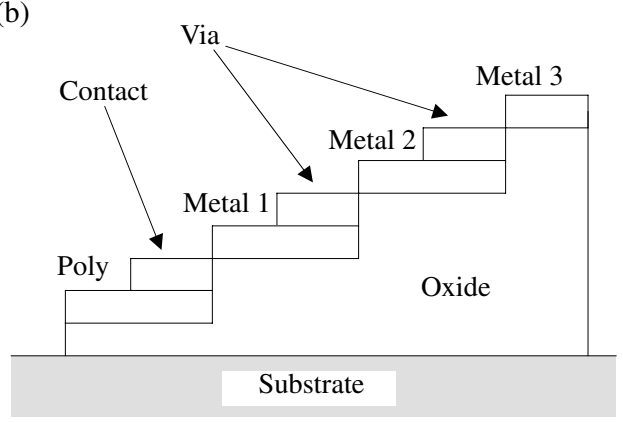

Figure 5. CMOS multi-level gratings: (a) four levels; $(b)$ eight levels. 
(a)

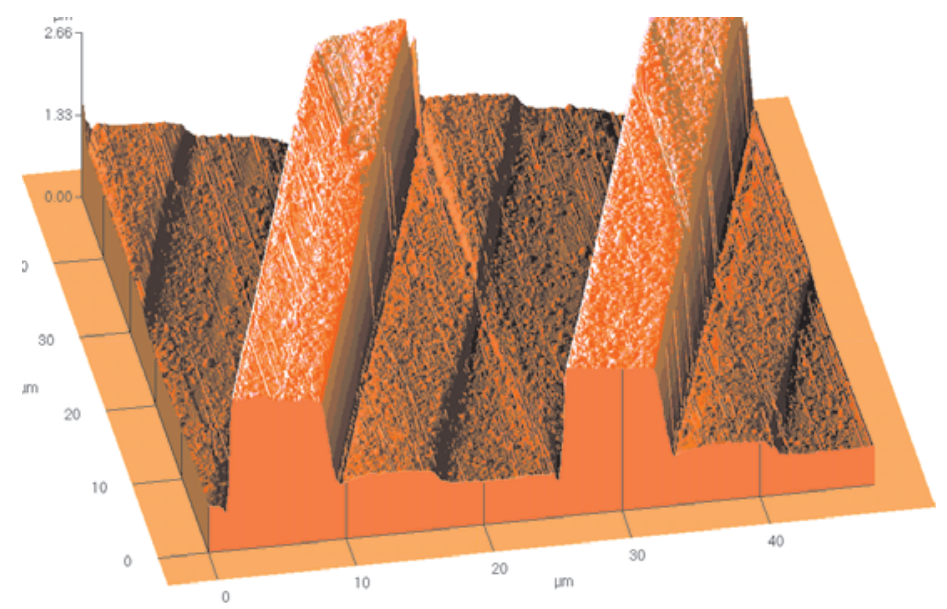

(b)

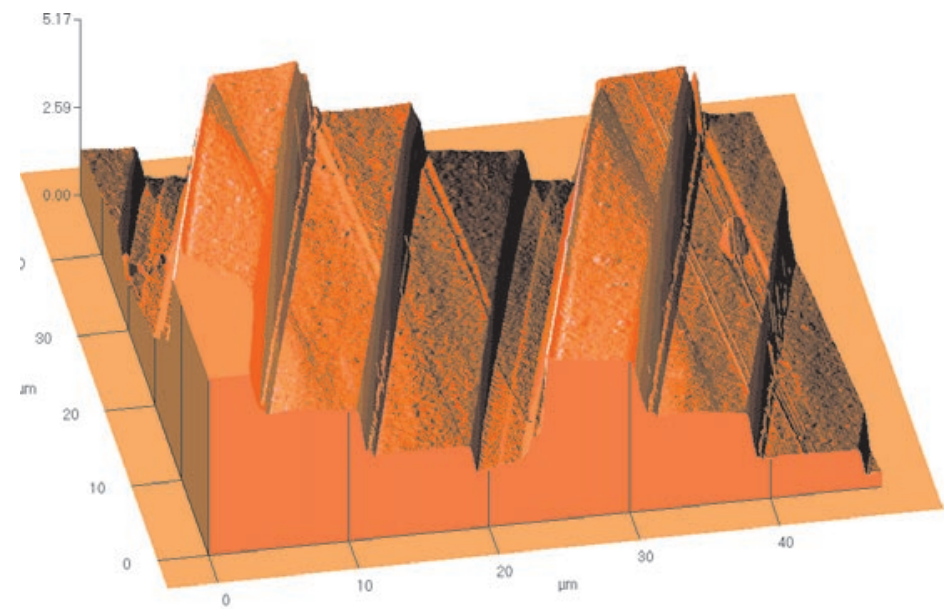

Figure 6. Atomic force microscopy (AFM) photographs of the CMOS four-phase-level gratings $(a)$ before and $(b)$ after silicon dioxide etching.

(This figure is in colour only in the electronic version)

the values of those built in GSOLVER, the derived optimum wavelength is $2.515 \mu \mathrm{m}$, which approximates to the result, $2.5 \mu \mathrm{m}$, computed by GSOLVER.

Summarizing this result, CMOS multi-level gratings have been proven to exhibit diffraction efficiencies up to $81.4 \%$ according to computational and theoretical analyses.

\section{Fabrication}

The multi-level gratings were fabricated by the conventional CMOS process and simple post-CMOS process. The fabrication follows commercial techniques and can be batchproduced immediately.

The single polysilicon three metal (SPTM) CMOS service of the Taiwan Semiconductor Manufacturing Company (TSMC) is employed to fabricate the multi-level gratings since an aluminium layer is a good etching mask for F-based plasma etching [13]. Figure 5(a) illustrates the cross section of the four-phase-level gratings. The silicon dioxide above the metal layers must be fully removed to expose those metal layers. Figure $6(a)$ shows the profile before the post-process, and

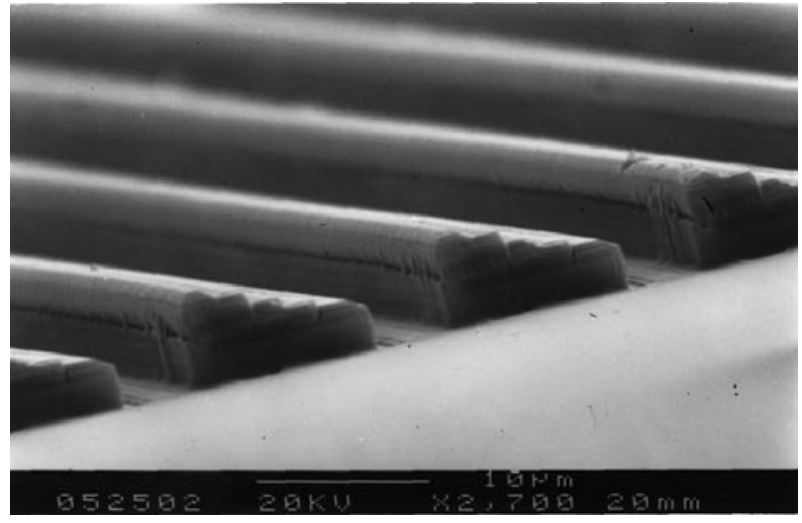

Figure 7. Scanning electron micrograph (SEM) of CMOS multi-level gratings.

shows metals 2 and 3 to be already exposed. The profile of metal 1 and the substrate, as shown in figure $6(b)$, is formed and the work is completed after silicon dioxide etching. Figure 7 shows an oblique view of multi-level gratings. 
Although the four-phase-level gratings were obtained by the standard CMOS process, the diffraction efficiency is insufficient (see figure 1). The attempt to design eight-phaselevel gratings was motivated by the need for higher diffraction efficiency. Figure 5(b) shows that the three metal layers, the poly, via and contact layers, are included to form the eight levels. The 'via' involves etching a tunnel in the silicon dioxide between two metal layers, such that the upper metal layer fills the via and drops down after deposition. However, the contact consists of tungsten deposited in the etching hole. The design of eight-phase-level gratings does not correspond to the CMOS rules, which require that the metal layer must be above the via or contact layers. The design still works, however, because the DOE is not an integrated circuit (IC) but an optical element. Finally, the CMOS gratings can be used directly as reflecting DOEs, or used as moulds in replication.

\section{Conclusions}

The four-phase-level gratings were fabricated according to the commercial CMOS process. The surface consisting of the metal layers was formed merely using silicon dioxide etching. The eight-phase-level gratings were designed for high diffraction efficiency. The criterion for the diffraction efficiency of multi-level gratings was determined and the design was shown to be extendable to other standard CMOS processes. CMOS multi-level gratings save development time and can be mass produced immediately since CMOS is a mature technology and a batch process. Furthermore, depth, alignment, dimension and shape errors can be reduced by advances in the CMOS process.

\section{Acknowledgments}

This work has been accomplished with much needed support and the authors wish to thank Professor C K Lee, Jennyi Chen, Hunghsuan Lin, Fuyuan Xiao, Tsungwei Huang, Shihchen Chang and Chunyuan Chi of the Institute of Applied Mechanics, National Taiwan University, for their valuable advice and assistance in the experiment.

\section{References}

[1] Swanson G J 1991 Binary optics technology: theoretical limits on the diffraction efficiency of multilevel diffractive optical elements Technology Report Lincoln Laboratory, Massachusetts Institute of Technology
[2] Chang M F and Wu M C 1998 Surface micromachined devices for microwave and photonic application Proc. SPIE ( Taiwan, ROC, July 1998) vol 3419 pp 214-68

[3] Stern M B 1997 Pattern transfer for diffractive and refractive microoptics Microelectron. Eng. 34299

[4] Däschner W, Long P, Larsson M and Lee S H 1995 Fabrication of diffractive optical elements using a signal optical exposure with a gray level mask J. Vac. Sci. Technol. B 132729

[5] Rothuizen H, Prongué D, Vasey F and Vettiger P 1997 A conic primitive-based pattern generator for e-beam lithography of diffractive optical elements Microelectron. Eng. 34243

[6] Kley E B 1997 Continuous profile writing by electron and optical lithography Microelectron.Eng. 34261

[7] Fan J, Zaleta D, Urquhart K S and Lee S H 1995 Efficiency encoding algorithms for computer-aided design of diffractive optical elements by the use of e-beam fabrication Appl. Opt. 342522

[8] Moldovan N, Dumbravescu N, Danlia M, Vitriuc A, Sindile P and Mohi J 1997 LIGA and alternative techniques for microoptical components CAS'97: Proc. Annual Semiconductor Conference (Sinaia, Romania, Oct. 2001) pp 149-52

[9] Socher E, Bochobza-Degani O and Nemirovsky Y 2001 A novel spiral CMOS compatible micromachined thermoelectric IR microsensor J. Micromech. Microeng. 11 574

[10] Berney H, Hill M, Cotter D, Hynes E, O’Neill M and Lane W A 2001 Determination of the effect of processing steps on the CMOS compatibility of a surface micromachined pressure sensor J. Micromech. Microeng. 11 402

[11] Robadey J, Paul O and Baltes H 1995 Two-dimensional integrated gas flow sensors by CMOS IC technology J. Micromech. Microeng. 5243

[12] Boltshauser T, Schonholzer M, Brand O and Baltes H 1992 Resonant humidity sensors using industrial CMOS-technology combined with post-processing J. Micromech. Microeng. 2205

[13] Dai C L, Chen H L and Chang P Z 2001 Fabrication of a micromachined optical modulator using the CMOS process J. Micromech. Microeng. 11612

[14] Yang L J, Huang T W and Chang P Z 2001 CMOS microelectromechanical bandpass filters Sensors Actuators A 90148

[15] Ehmann I M, Ruther P, von Arx M and Paul O 2001 Operation and short-term drift of polysilicon-heated CMOS microstructures at temperatures up to $1200 \mathrm{~K}$ J. Micromech. Microeng. 11397

[16] Gianchandani Y B, Kim H, Shinn M, Ha B, Lee B, Najafi K and Song C $2000 \mathrm{~A}$ fabrication process for integrating polysilicon microstructures with post-processed CMOS circuits J. Micromech. Microeng. 10380

[17] Mayer M, Paul O and Baltes H 1998 Complete set of piezoresistive coefficients of CMOS $\mathrm{n}^{+}$diffusion J. Micromech. Microeng. 8158

[18] Lang M and Glesner M 1997 An acceleration sensor in CMOS-compatible technology for integration in complex systems J. Micromech. Microeng. 7193 